\title{
Diagnosis of Multiple Sclerosis in a Previously Asymptomatic Woman After Ruptured Ectopic Pregnancy and Cardiac Arrest
}

\author{
Venkatachalam Veerappan ${ }^{\mathrm{a}}$, Anthony DiCamillo ${ }^{\mathrm{a}, \mathrm{b}}$
}

\begin{abstract}
This case report recounts the sequence of events of a previously healthy 39-year-old female who was diagnosed with multiple sclerosis (MS) after presenting with cardiopulmonary arrest after ectopic pregnancy. Additionally, various pertinent aspects of this case are discussed here, both in regards to pregnancy and MS and with the radiological findings in asymptomatic individuals. MS is a chronic disease of the brain and spinal cord which is autoimmune in nature, although the full etiology is still unknown. The symptoms of the disease are numerous and varied including more commonly vision loss, numbness and weakness. The disease is often initially diagnosed between ages 20 and 40 years, but many patients first present with attacks earlier or later in life. Currently the diagnosis is based much on clinical attacks and MRI results. There is no cure at this time for MS; however, there are many current therapies which are available to decrease disease activity.
\end{abstract}

Keywords: Multiple sclerosis; Pregnancy; Radiologically isolated syndrome

\section{Introduction}

Multiple sclerosis (MS) is an inflammatory disease of the central nervous, brain and spinal cord, in which myelin and axons are damaged by lymphocytic infiltration [1]. First of note are some general demographics in regards to MS. The average age of onset is 30 and the disease appears to affect females at least twice as much as compared to males. It is a relatively common disorder affecting nearly 400,000 people in the United States and an estimated 2.5 million worldwide

Manuscript accepted for publication August 13, 2014

${ }^{a}$ Neurology Department, Valley Hospital Medical Center, Las Vegas, NV, USA

${ }^{\mathrm{b}}$ Corresponding Author: Anthony DiCamillo, 2020 Goldring Avenue Suite 202, Las Vegas, NV 89106, USA. Email: dicamill@nova.edu

doi: http://dx.doi.org/10.14740/jnr286w
[1, 2-5]. Although MS is a fairly common disease the case report here describes a very unique presentation of the disease, which does not appear to have ever been reported in the literature. In this case the patient, who presented with cardiac arrest from a ruptured ectopic pregnancy subsequently through the completion of her workup, was diagnosed with MS. After the case presentation there is a discussion of the pertinent aspects of the case.

\section{Case Report}

A 39-year-old right-handed Caucasian female presented with an unresponsive episode. Prior to presentation to the hospital the patient was noted to be having increasing abdominal and flank pain over $24 \mathrm{~h}$. The pain was becoming more severe and so the patient was in the process of going to the hospital. The patient's husband had left their home to take their children to the grandmother's home. When the patient's husband returned home, he found the patient on the floor and she was unresponsive. EMS was contacted. There was no report of CPR being performed prior to EMS arrival. Upon EMS arrival, the patient was found to be in asystole and ALCS/CPR was started. After 15 min of ACLS, the patient did have a return of spontaneous circulation for $5 \mathrm{~min}$. However, she subsequently went on to have a second cardiac arrest in which she was found to be in pulseless electrical activity. The second arrest lasted for approximately $20 \mathrm{~min}$. The patient was intubated in the field prior to arrival at the emergency department.

During her emergency department course the patient regained spontaneous circulation and a "code chill" (hypothermic protocol) was started. However, the code chill was cancelled, due to a pending surgery. The patient's initial laboratory data showed a positive pregnancy test; therefore, a bedside pelvic ultrasound was preformed. Results of the ultrasound showed a ruptured ectopic pregnancy with a moderate amount of free fluid in the abdomen and pelvis. The gestational age was estimated at 9 weeks and 6 days. OBGYN consultation was immediately obtained from the emergency department and the patient was then taken from the ER to the OR for emergent surgery. During the surgery, the OBGYN estimated that between 1,500 to $2,000 \mathrm{~mL}$ of 

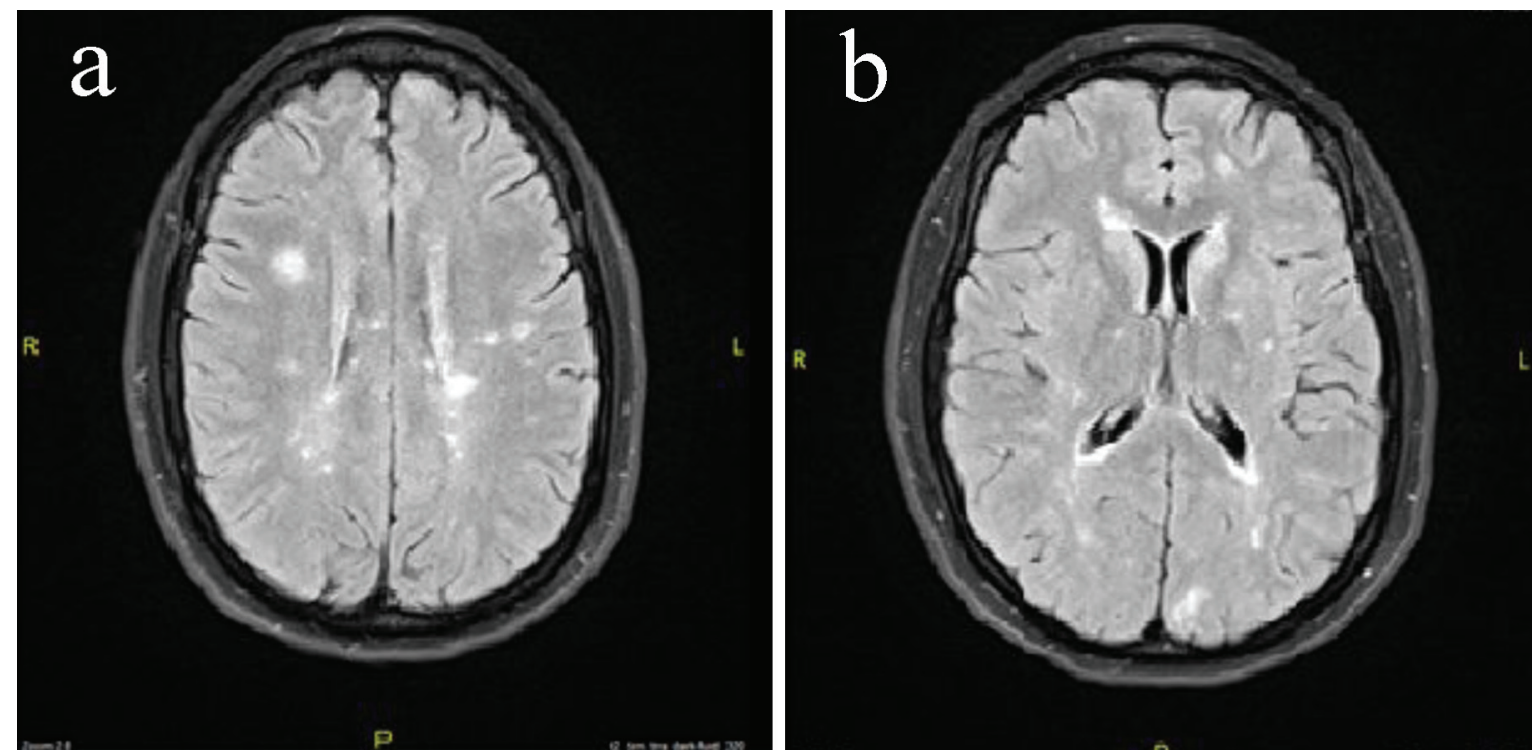

Figure 1. (a) MRI of the brain without contrast axial images of the patient showing multiple periventricular lesions. (b) MRI of the brain without contrast axial images of the patient showing juxtacortical and periventricular lesions.

blood and blood clots were removed from the pelvis. After the surgery the patient was then transferred to ICU care in a guarded condition.

Further past medical history was obtained from the husband. Previously the patient was in good medical condition and took no regular prescribed medications. She had allergies to demeral and darvocet. The patient had had three previous pregnancies which included one cesarean section. She was a previous smoker, but currently was a non-smoker. There was no illegal drug use reported and only occasionally alcohol use. She had no significant or contributory family history.

After surgery, while the patient was intubated and under ICU status, neurological consultation was requested. The request was in regards to possible anoxic or hypoxic encephalopathy resulting from prolonged cardiopulmonary arrest time for at least $35 \mathrm{~min}$. During the first physical examination, the patient was intubated and was on sedative medications. Consequently the physical and neurological exam findings were limited. Full testing in regards to her encephalopathy was ordered at that time including: EEG to
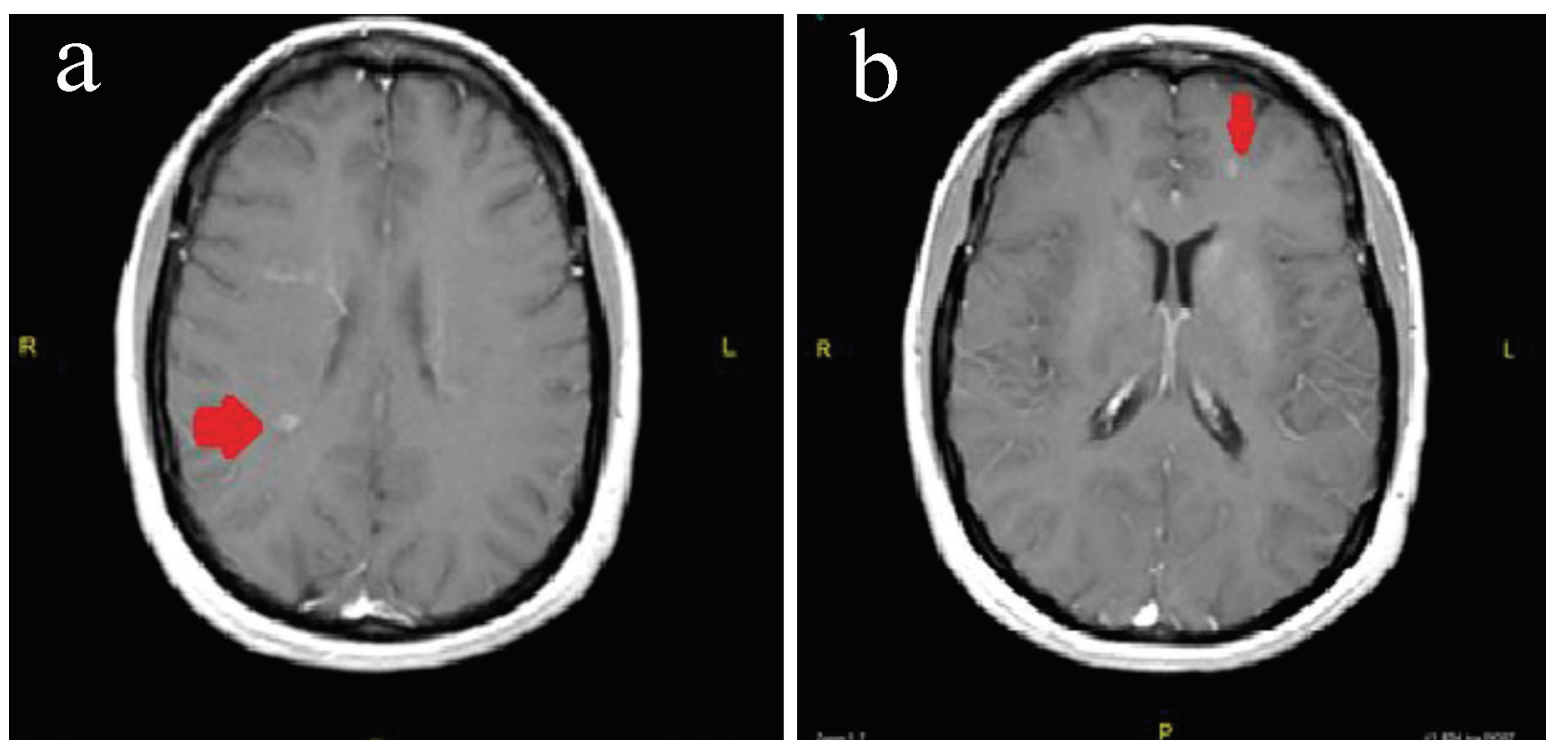

Figure 2. (a) MRI of the brain with and without contrast showing a right-sided periventricular enhancing lesion. (b) MRI of the brain with and without contrast showing a left-side juxtacortical frontal lobe enhancing lesions. 


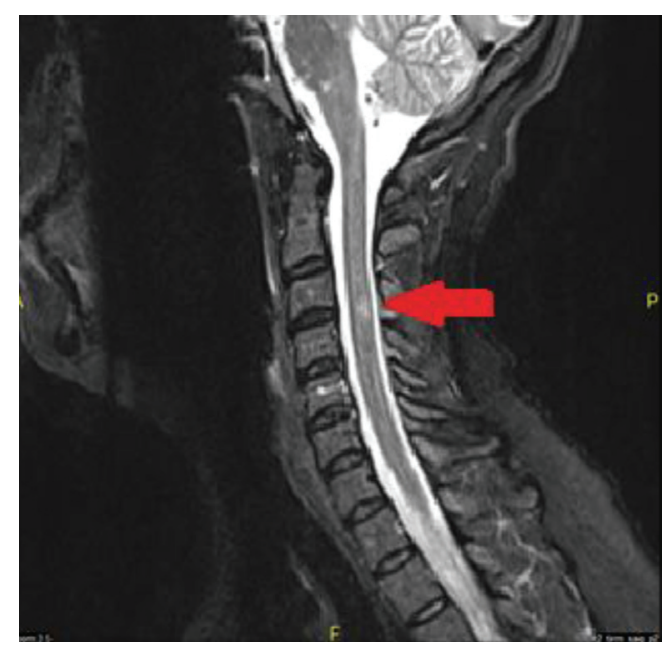

Figure 3. Cervical spine MRI with and without. Lesion present without contrast enhancement.

evaluate for any subclinical seizure activity, MRI of the brain to assess for stroke or cerebra edema, and standard metabolic labs as well.

During her second day in the ICU, the patient was weaned off sedation and began to become more awake and alert. She was shortly thereafter able to be extubated. The EEG prior to extubation showed only a slight slowing of the background, no seizure activity or other pathologic findings. After extubation, the patient had no complaints; however, upon more in-depth questioning she did have some mild memory loss of the event and for a short period of time prior to the event. The patient's physical and neurological examination at that time, were essentially otherwise unremarkable and she was back to normal regarding motor strength, sensation and higher mental function. However, testing of her memory did indicate that the patient had some mild difficulty with short-term recall. She could only recall two out of three words at $5 \mathrm{~min}$ and could not recall the third word even with multiple cues.

Although the MRI scan had been ordered prior to extubation to evaluate for any injury related to the cardiac arrest, the MRI of the brain without contrast was not completed until after the patient had been extubated. The findings showed no acute stroke; however, unexpectedly there were numerous foci of flair and T2 hyperintensities. Lesions were present both periventricular and juxtacortical consistent with demyelination areas that are present in MS (Fig. 1a, b).

The patient strongly denied history of any neurological events previously in her life. Again at this time she had no objective clinical signs of MS. At this time further workup was set to be carried out in the outpatient setting. At this time her case was initially thought to be one of a radiologically isolated syndrome (RIS).

Three days later the patient was doing well and participating with therapy on the medical-surgical floor, when an

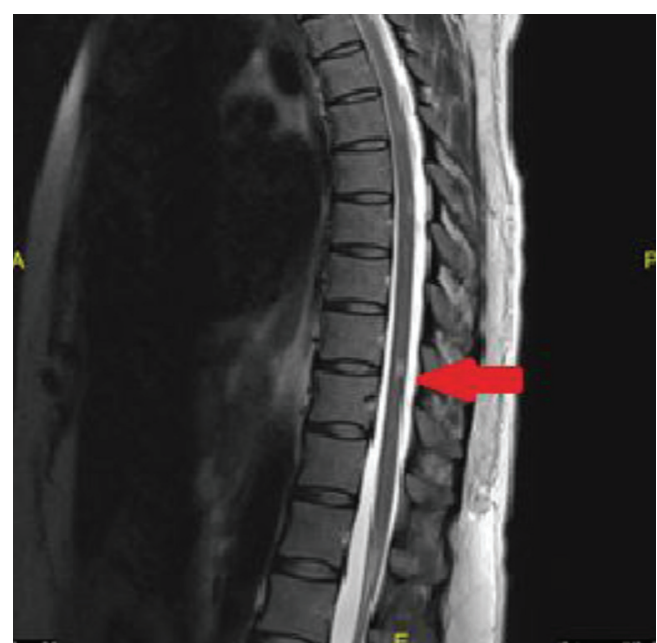

Figure 4. Thoracic spine MRI showing a lesion which did demonstrate contrast enhancement.

unexpected change occurred. The patient had new onset of psychosis with both auditory and visual hallucinations. The patient became paranoid, agitated, aggressive and confused. The patient's family was questioned and they strongly denied any history of mental disorder or psychosis in the past. Psychiatry was consulted and the patient was also placed on antipsychotic medication. A repeat EEG was ordered during this time and it consequently showed sharp and sharp-wave activity that lasted from 1 to up to $3 \mathrm{~s}$ mainly over the right frontotemporal region. It was decided then to order a repeat MRI of the brain, this time with contrast. The patient was also placed on antiepileptic medications, and during the next several days the patient's mood and mentation began to improve.

Results of MRI of the brain with and without contrast showed similar findings to her previous MRI of the brain; however, the contrast scan did show multiple areas of enhancement consistent with active demyelination. Some of the larger lesions included an active lesion in the left frontal lobe as well as the right parietal lobe (Fig. 2a, b). Throughout her stay in the hospital she did not have any other clinical symptoms of demyelination with the exception of two complaints. One day she stated that she had numbness involving the right hand and fingers. However, she was still altered from her baseline when she expressed that she had the paresthesias of the right hand. Additionally she did complain of decreased visual acuity more in the left eye compared to the right and this complaint did persist even after her psychosis had resolved; however, the vision did improve over a period of several days.

The decision to start treatment and to pursue further testing was made after the results of the contrasted MRI of the brain were reviewed. The patient was still having mood disturbances at the time that the second MRI of the brain was completed and so it was decided to avoid steroid treatment 
as there was concern that steroids could possibly worsen the psychosis. The patient was started on IVIG once a day for 3 days. During that time a screening MRI of the cervical spine and thoracic spine was ordered to evaluate for any demyelinating lesions. The cervical spine MRI showed two areas consistent with demyelination without contrast enhancement (Fig. 3). The thoracic spine, however, not only showed evidence of chronic demyelination but also showed active contrast enhancement (Fig. 4). The patient remained completely asymptomatic despite the location of all of these lesions, and there are no paresthesias of her trunk or lower extremities and also no weakness in the lower extremities.

The patient continued to improve clinically with regard to her paranoid and psychosis. By the completion of her 3-day course of IVIG, she had essentially returned back to her baseline function. It was recommended to her that she should have a lumbar puncture done to evaluate her CSF for various tests; however, she refused at that time. Blood work was sent out for autoimmune processes and was significant for mildly elevated Sjogen's SS-A antibody at a level of 1.1 (normal range 0.0 - 0.9) and mildly elevated anti-Smith antibody at 26 (normal range 0 - 19). However, all other autoimmune workup including ANA as well as double stranded DNA were negative. In addition to the autoimmune labs the patient had a low vitamin D-25 hydroxy level at 17.7. The patient was discharged home to her family in stable condition. She was giving information regarding follow-up in the clinic to discuss further workup and treatment. The patient was in agreement with follow-up.

\section{Discussion}

There is an established association between ectopic pregnancy and maternal cardiac arrest. Ectopic pregnancy occurs when a developing blastocyst becomes implanted at a site other than the endometrium within the uterine cavity. The overall incidence of ectopic pregnancy increased during the middle of the 20th century. Most recent approximations are that about 20 per 1,000 or $2 \%$ of all pregnancies are considered ectopic [6]. The classic symptoms in an ectopic pregnancy include abdominal pain, amenorrhea and vaginal bleeding. These symptoms can occur in both ruptured and unruptured cases [7]. When ruptured ectopic leads to severe blood loss, it can cause cardiac arrest and death. Most of the deaths, approximately $85 \%$, associated with ectopic pregnancy are due to blood loss. In the United States from 1998 to $2005,4 \%$ of all maternal deaths occurred secondary to ectopic pregnancies complications. Ectopic pregnancy is the most common cause of first trimester pregnancy related death in the United States. Recently, an earlier diagnosis and treatment have dramatically decreased the number of deaths due to ectopic pregnancy [8].

There are known links between MS and pregnancy. In regard to pregnancies in patients with MS, a study has shown that women with MS do not have an increased number of delivery complications, stillbirths, children with birth defects, preterm births, spontaneous abortions or ectopic pregnancies as compared to general population [9-13]. Although there have previously been disagreements, the data now have shown that pregnancy is associated with a significant decrease in MS disease activity. In contrast, the postpartum period is associated with an increase in MS activity $[14,15]$. The data from the pregnancy in MS study (PRIMS) have one of the best individual data regarding the association of decreased disease activity during pregnancy and increased activity after the birth. In the PRIMS study, there were a total of 254 women with MS who were followed for a period of 2 years. They were followed not only during their pregnancies, but also for 12 months postpartum. The average rates of relapse before pregnancy, in the first trimester, in the second trimester, in the third trimester, and in the first 3 months postpartum were respectively $0.7,0.5,0.6,0.2$ and 1.2 per woman per year. The factors which lead to relapses after the first 3 months after giving birth included increased relapse rate in the year before pregnancy, increased relapse rate during pregnancy and higher EDSS scores at the onset of pregnancy. Even with the rise in relapses rating in the first few months postpartum, it should be noted that in the long term there has not been acceleration in the rate of disability or disease progression postpartum [16-18]. While no formal studies have reviewed cases such as the patient discussed above here, inference could be made that the lost of the fetus by ruptured ectopic would increase MS activity just as the patients studied in a true postpartum period.

The patient, if granted that her visual symptoms or paresthesias are labeled as a clinic attack, has by the most recent diagnostic criteria definitive MS. The McDonald criteria, which was developed, in 2001, and subsequently revised in 2005 and in 2010 guides the diagnosis based on the facts that this patient has both dissemination of disease in time and in space. Dissemination in time is demonstrated if an MRI has both gadolinium-enhancing and non-enhancing lesions at any one time, which was present in our case. Dissemination in space is demonstrated when the MRI has T2 lesions present in at least two of the four regions which are noted as MS-typical areas of the CNS. These regions are periventricular, juxtacortical, infratentorial and spinal cord. The patient in the case report again had multiple lesions of all the above mentioned areas. Additionally it should be noted that an MS attack or exacerbation is defined within the McDonald criteria as patient-reported or objective event which is typical of an acute inflammatory demyelinating event in the CNS. It must by definition last at least $24 \mathrm{~h}$ and should not occur in the setting of a fever or other infection [19-24]. The exacerbation in the definition should then be confirmed by a neurologic examination, if possible. Before a definite diagnosis of MS is made, the patient must have had at least one attack that 
is confirmed by findings on a neurologic examination, visual evoked potential response testing in setting of visual complaint or with MRI results that would be consistent with the prior noted neurologic symptoms [25-28]. In the case report above, visual evoked response testing would be pertinent in giving further substantial evidence that the patient does indeed have MS.

Initially there was discussion of the potential of this being a case of RIS as the patient's clinic symptoms were not yet present after initial MRI was complete. The clinic picture was not in correspondence with the present radiological findings. Especially of note were the active yet asymptotic thoracic spine cord lesions seen on this patient's thoracic spine MRI. RIS is a relatively new phenomenon first described in 2009 by Okuda [29]. RIS is typically found when MRIs are being ordered for other purposes especially headaches or other conditions. In our presented case, the patient's MRI was ordered to evaluate for brain injury after cardiopulmonary arrest. The initial proposed diagnostic criteria for RIS include the presence of incidentally identified CNS white matter anomalies meeting the MRI criteria of being ovoid, well-circumscribed and homogeneous with or with involvement of the corpus callousm. Also there must be T2 hyperintensities measuring greater than $3 \mathrm{~mm}$ and also fulfilling Barkhof criteria for dissemination in space. The CNS white matter lesions must not be consistent with a vascular pattern. There additionally has to be no history of remitting clinical symptoms consistent with neurologic dysfunction. Further requirements also include that the CNS anomalies seen on MRI are not explained better by another disease [30].

The data from the early evaluation by Okuda et al showed that patients with RIS at an average time of 5.4 years, with a range of $1.1-9.8$, went on to develop clinically isolated syndrome (CIS) of MS. Progression of radiological finding including T2 foci, enlarging of foci or contrast enhancing lesions occurred in $59 \%$ of patient's at a median time of 2.7 years. During this study it was found that if patient's had contrast enhancing lesions on the first MRI indentifying RIS, there was a significant risk of going on to have dissemination in time on future MRIs of the brain [31]. Contrast enhancing lesions have been found to be a prognosticator of progression radiologically and subsequent clinical relapses. Also it is the most predictive MRI finding in predicting the development into clinically defined MS [32-33]. An additional important piece of literature by Okuda et al in the RIS field evaluated further asymptomatic spinal cord lesions and progression of the disease. In the study, 71 patients had cervical MRIs done prior to the development of any clinical events, 17 of the 71 had thoracic spine MRIs. Out of the 71 subjects 25 (35\%) were found to have anomalies very suggestive of demyelination prior to any clinical progression. Of those patients, six had scans which showed contrast enhancing lesions. Of the patients which had thoracic MRIs performed, six of the 17 had lesions present and five of the six also had cervical spine disease noted. The disease progressed to CIS or a primary progression MS in 21 out of those 25 patients, or $84 \%$. The average time to clinical progression of the disease was 1.6 years [34]. The importance of the study overall and in regard to the case report presented here is that the results suggest that patients with asymptomatic disease within the brain and spinal cord in comparison to individuals that only have RIS in the brain are at extremely high risk for going on to develop clinical symptoms [35-38].

There are many interesting facets to this case. The first aspect was that the patient survived such a prolonged period of cardiopulmonary arrest and recovered neurologically intact. Second, is the fact that there were multiple contrast enhancing lesions on the brain and the thoracic spine MRIs yet the patient appeared generally asymptotic from them. In an extensive search of literature there does not appear to be any cases of MS found incidentally after cardiopulmonary arrest and also no reported cases of severely active MS after ruptured ectopic pregnancy. Although there is sufficient information available regarding MS in the natural postpartum period, information regarding disease activity after miscarriages and ectopic pregnancies is still incomplete and lacking at this point. Further information on this topic would help both neurologists and patients determine the best course of action, if and when the situation occurs.

\section{References}

1. Compston A, Coles A. Multiple sclerosis. Lancet. 2008;372(9648):1502-1517.

2. World Health Organization. Atlas: Multiple Sclerosis Resources in the World 2008. Geneva: World Health Organization. 2008. pp. 15-16. ISBN 92-4-156375-3.

3. Alonso A, Hernan MA. Temporal trends in the incidence of multiple sclerosis: a systematic review. Neurology. 2008;71(2):129-135.

4. Koch-Henriksen N, Sorensen PS. The changing demographic pattern of multiple sclerosis epidemiology. Lancet Neurol. 2010;9(5):520-532.

5. Dunn SE, Steinman L. The gender gap in multiple sclerosis: intersection of science and society. JAMA Neurol. 2013;70(5):634-635.

6. Ectopic pregnancy--United States, 1990-1992. Centers for Disease Control and Prevention (CDC) MMWR Morb Mortal Wkly Rep. 1995;44(3):46.

7. Ankum WM, Mol BW, Van der Veen F, Bossuyt PM. Risk factors for ectopic pregnancy: a meta-analysis. Fertil Steril. 1996;65(6):1093-1099.

8. Berg CJ, Callaghan WM, Syverson C, Henderson Z. Pregnancy-related mortality in the United States, 1998 to 2005. Obstet Gynecol. 2010;116(6):1302-1309.

9. Mueller BA, Zhang J, Critchlow CW. Birth outcomes and need for hospitalization after delivery among 
women with multiple sclerosis. Am J Obstet Gynecol. 2002;186(3):446-452.

10. Confavreux C, Hutchinson M, Hours MM, CortinovisTourniaire P, Moreau T. Rate of pregnancy-related relapse in multiple sclerosis. Pregnancy in Multiple Sclerosis Group. N Engl J Med. 1998;339(5):285-291.

11. Worthington J, Jones R, Crawford M, Forti A. Pregnancy and multiple sclerosis--a 3-year prospective study. J Neurol. 1994;241(4):228-233.

12. Houtchens MK. Pregnancy and multiple sclerosis. Semin Neurol. 2007;27(5):434-441.

13. Runmarker B, Andersen O. Pregnancy is associated with a lower risk of onset and a better prognosis in multiple sclerosis. Brain. 1995;118 ( Pt 1):253-261.

14. Finkelsztejn A, Brooks JB, Paschoal FM, Jr., Fragoso YD. What can we really tell women with multiple sclerosis regarding pregnancy? A systematic review and meta-analysis of the literature. BJOG. 2011;118(7):790797.

15. Verdru P, Theys P, D’Hooghe MB, Carton H. Pregnancy and multiple sclerosis: the influence on long term disability. Clin Neurol Neurosurg. 1994;96(1):38-41.

16. Vukusic S, Hutchinson M, Hours M, Moreau T, Cortinovis-Tourniaire P, Adeleine P, Confavreux C, et al. Pregnancy and multiple sclerosis (the PRIMS study): clinical predictors of post-partum relapse. Brain. 2004;127(Pt 6):1353-1360.

17. Franklin GM, Tremlett H. Multiple sclerosis and pregnancy: what should we be telling our patients? Neurology. 2009;73(22):1820-1822.

18. Dahl J, Myhr KM, Daltveit AK, Hoff JM, Gilhus NE. Pregnancy, delivery, and birth outcome in women with multiple sclerosis. Neurology. 2005;65(12):1961-1963.

19. McDonald WI, Compston A, Edan G, Goodkin D, Hartung HP, Lublin FD, McFarland HF, et al. Recommended diagnostic criteria for multiple sclerosis: guidelines from the International Panel on the diagnosis of multiple sclerosis. Ann Neurol. 2001;50(1):121-127.

20. Polman CH, Reingold SC, Edan G, Filippi M, Hartung HP, Kappos L, Lublin FD, et al. Diagnostic criteria for multiple sclerosis: 2005 revisions to the "McDonald Criteria”. Ann Neurol. 2005;58(6):840-846.

21. Polman CH, Reingold SC, Banwell B, Clanet M, Cohen JA, Filippi M, Fujihara K, et al. Diagnostic criteria for multiple sclerosis: 2010 revisions to the McDonald criteria. Ann Neurol. 2011;69(2):292-302.

22. Swanton JK, Rovira A, Tintore M, Altmann DR, Barkhof F, Filippi M, Huerga E, et al. MRI criteria for multiple sclerosis in patients presenting with clinically isolated syndromes: a multicentre retrospective study. Lancet Neurol. 2007;6(8):677-686.

23. Rovira A, Swanton J, Tintore M, Huerga E, Barkhof F, Filippi M, Frederiksen JL, et al. A single, early magnetic resonance imaging study in the diagnosis of multiple sclerosis. Arch Neurol. 2009;66(5):587-592.

24. Montalban X, Tintore M, Swanton J, Barkhof F, Fazekas F, Filippi M, Frederiksen J, et al. MRI criteria for MS in patients with clinically isolated syndromes. Neurology. 2010;74(5):427-434.

25. Bot JC, Barkhof F, Polman CH, Lycklama a Nijeholt GJ, de Groot V, Bergers E, Ader HJ, et al. Spinal cord abnormalities in recently diagnosed MS patients: added value of spinal MRI examination. Neurology. 2004;62(2):226233.

26. Filippi M. Enhanced magnetic resonance imaging in multiple sclerosis. Mult Scler. 2000;6(5):320-326.

27. Rovira A, Alonso J, Cucurella G, Nos C, Tintore M, Pedraza S, Rio J, et al. Evolution of multiple sclerosis lesions on serial contrast-enhanced T1-weighted and magnetization-transfer MR images. AJNR Am J Neuroradiol. 1999;20(10):1939-1945.

28. Lee KH, Hashimoto SA, Hooge JP, Kastrukoff LF, Oger JJ, Li DK, Paty DW. Magnetic resonance imaging of the head in the diagnosis of multiple sclerosis: a prospective 2-year follow-up with comparison of clinical evaluation, evoked potentials, oligoclonal banding, and CT. Neurology. 1991;41(5):657-660.

29. Okuda DT, Mowry EM, Beheshtian A, Waubant E, Baranzini SE, Goodin DS, Hauser SL, et al. Incidental MRI anomalies suggestive of multiple sclerosis: the radiologically isolated syndrome. Neurology. 2009;72(9):800805.

30. Barkhof F, Filippi M, Miller DH, Scheltens P, Campi A, Polman CH, Comi G, et al. Comparison of MRI criteria at first presentation to predict conversion to clinically definite multiple sclerosis. Brain. 1997;120 ( Pt 11):2059-2069.

31. Barkhof F, Held U, Simon JH, Daumer M, Fazekas F, Filippi M, Frank JA, et al. Predicting gadolinium enhancement status in MS patients eligible for randomized clinical trials. Neurology. 2005;65(9):1447-1454.

32. Kermode AG, Thompson AJ, Tofts P, MacManus DG, Kendall BE, Kingsley DP, Moseley IF, et al. Breakdown of the blood-brain barrier precedes symptoms and other MRI signs of new lesions in multiple sclerosis. Pathogenetic and clinical implications. Brain. 1990;113 ( Pt 5):1477-1489.

33. Kappos L, Moeri D, Radue EW, Schoetzau A, Schweikert K, Barkhof F, Miller D, et al. Predictive value of gadolinium-enhanced magnetic resonance imaging for relapse rate and changes in disability or impairment in multiple sclerosis: a meta-analysis. Gadolinium MRI Meta-analysis Group. Lancet. 1999;353(9157):964-969.

34. Koudriavtseva T, Thompson AJ, Fiorelli M, Gasperini C, Bastianello S, Bozzao A, Paolillo A, et al. Gadolinium enhanced MRI predicts clinical and MRI disease activity in relapsing-remitting multiple sclerosis. J Neurol Neurosurg Psychiatry. 1997;62(3):285-287. 
35. Okuda DT, Mowry EM, Cree BA, Crabtree EC, Goodin DS, Waubant E, Pelletier D. Asymptomatic spinal cord lesions predict disease progression in radiologically isolated syndrome. Neurology. 2011;76(8):686-692.

36. Poser CM, Paty DW, Scheinberg L, McDonald WI, Davis FA, Ebers GC, Johnson KP, et al. New diagnostic criteria for multiple sclerosis: guidelines for research protocols. Ann Neurol. 1983;13(3):227-231.
37. Offenbacher H, Fazekas F, Schmidt R, Freidl W, Flooh E, Payer F, Lechner H. Assessment of MRI criteria for a diagnosis of MS. Neurology. 1993;43(5):905-909.

38. Paty DW, Oger JJ, Kastrukoff LF, Hashimoto SA, Hooge JP, Eisen AA, Eisen KA, et al. MRI in the diagnosis of MS: a prospective study with comparison of clinical evaluation, evoked potentials, oligoclonal banding, and CT. Neurology. 1988;38(2):180-185. 